\title{
Ultrafast hydrogen bond dynamics of liquid water revealed by terahertz-induced transient birefringence
}

\author{
Hang Zhao', Yong Tan', Liangliang Zhang ${ }^{2}$, Rui Zhang ${ }^{3}$, Mostafa Shalaby ${ }^{2}$, Cunlin Zhang ${ }^{2}$, Yuejin Zhao ${ }^{1}$ and \\ Xi-Cheng Zhang (1) ${ }^{4}$
}

\begin{abstract}
The fundamental properties of water molecules, such as their molecular polarizability, have not yet been clarified. The hydrogen bond network is generally considered to play an important role in the thermodynamic properties of water. The terahertz $(\mathrm{THz})$ Kerr effect technique, as a novel tool, is expected to be useful in exploring the low-frequency molecular dynamics of liquid water. Here, we use an intense and ultrabroadband THz pulse (peak electric field strength of $14.9 \mathrm{MV} / \mathrm{cm}$, centre frequency of $3.9 \mathrm{THz}$, and bandwidth of $1-10 \mathrm{THz}$ ) to resonantly excite intermolecular modes of liquid water. Bipolar THz field-induced transient birefringence signals are observed in a free-flowing water film. We propose a hydrogen bond harmonic oscillator model associated with the dielectric susceptibility and combine it with the Lorentz dynamic equation to investigate the intermolecular structure and dynamics of liquid water. We mainly decompose the bipolar signals into a positive signal caused by hydrogen bond stretching vibration and a negative signal caused by hydrogen bond bending vibration, indicating that the polarizability perturbation of water presents competing contributions under bending and stretching conditions. A Kerr coefficient equation related to the intermolecular modes of water is established. The ultrafast intermolecular hydrogen bond dynamics of water revealed by an ultrabroadband $\mathrm{THz}$ pump pulse can provide further insights into the transient structure of liquid water corresponding to the pertinent modes.
\end{abstract}

\section{Introduction}

Liquid water is considered the cornerstone of life and has many extraordinary physical and biochemical properties $^{1,2}$. The hydrogen bond network of liquid water is widely recognized to play a crucial role in these properties $^{3-11}$. The dielectric susceptibility $\chi(\omega)$ reflects the collective and/or cooperative molecular motions with

Correspondence: Liangliang Zhang (zhlliang@126.com) or

Yuejin Zhao (yjzhao@bit.edu.cn)

${ }^{1}$ Beijing Key Laboratory for Precision Optoelectronic Measurement Instrument and Technology, School of Optics and Photonics, Beijing Institute of Technology, Beijing 100081, China

${ }^{2}$ Beijing Advanced Innovation Center for Imaging Technology and Key Laboratory of Terahertz Optoelectronics (MoE), Department of Physics, Capital Normal University, Beijing 100048, China

Full list of author information is available at the end of the article

These authors contributed equally: Hang Zhao, Yong Tan frequencies from the gigahertz $(\mathrm{GHz})$ to terahertz $(\mathrm{THz})$ range and is strongly influenced by the dynamics of intermolecular modes ${ }^{12} \cdot \chi(\omega)$ can be evaluated by various techniques, such as far-infrared spectroscopy ${ }^{13,14}$, lowfrequency Raman spectroscopy ${ }^{15,16}, \mathrm{THz}$ time-domain spectroscopy $^{17,18}$ and 2D Raman-THz spectroscopy ${ }^{19}$, which provide insights into the structure, stability and rearrangement dynamics of the hydrogen bond network. However, the determination of the molecular motions that make the dominant contributions to $\chi(\omega)$ is difficult because the complexity of intermolecular interactions and the large spectral overlap of relevant modes, such as relaxation processes and intermolecular modes, make the assignment of the underlying dynamics challenging.

The time-resolved optical Kerr effect (OKE) technique $^{20-23}$ is a powerful experimental tool enabling accurate

\section{(c) The Author(s) 2020}

(c) (i) Open Access This article is licensed under a Creative Commons Attribution 4.0 International License, which permits use, sharing, adaptation, distribution and reproduction cc) in any medium or format, as long as you give appropriate credit to the original author(s) and the source, provide a link to the Creative Commons license, and indicate if changes were made. The images or other third party material in this article are included in the article's Creative Commons license, unless indicated otherwise in a credit line to the material. If material is not included in the article's Creative Commons license and your intended use is not permitted by statutory regulation or exceeds the permitted use, you will need to obtain permission directly from the copyright holder. To view a copy of this license, visit http://creativecommons.org/licenses/by/4.0/. 
investigation of dynamic phenomena in molecular liquids. However, since the rotation of a water molecule's permanent dipole moment and other low-frequency motion modes cannot follow the ultrafast electric field oscillation, the contribution of water molecular motions to $\chi(\omega)$ is much smaller than that of electron cloud distortion. Therefore, when the OKE technique is used to investigate intermolecular hydrogen bond motions, the birefringence signal is always positive, and the molecular response is extremely weak. An enhanced molecular response can be obtained when using a THz electric field, as the field is in resonance with the rotational transitions of a single molecule or the cooperative low-frequency molecular motions ${ }^{24-26}$. Therefore, the time-resolved $\mathrm{THz}$ Kerr effect (TKE) response is expected to be a powerful phenomenon for exploring the low-frequency dynamics of water molecules.

Recently, researchers have successfully used $\mathrm{THz}$ pulses to drive liquids, such as dimethyl sulfoxide (DMSO), chloroform and liquid water, and tracked the molecular dynamics by detecting transient birefringence ${ }^{24,27-33}$. In particular, in 2018, Zalden et al. used a low-frequency single-cycle $\mathrm{THz}$ pulse (centre frequency of $0.25 \mathrm{THz}$ ) generated by optical rectification in a $\mathrm{LiNbO}_{3}$ crystal to stimulate liquid water and observed the time-resolved birefringent signal induced by the transient orientation of dipole moments. They concluded that the polarizability of a water molecule parallel to the direction of the permanent dipole moment is smaller than the polarizability perpendicular to the permanent dipole moment ${ }^{32}$. However, only the polarizability anisotropy caused by the orientational relaxation of liquid water was investigated in this study, which is limited by the relatively low-power and narrowband properties of the applied $\mathrm{THz}$ excitation. In the latest research, Novelli et al. presented a narrowband $\mathrm{THz}$ pump/THz probe experiment using a free electron laser to induce strong anisotropy of liquid water through librational excitation ${ }^{33}$. A very large third-order response of liquid water at $12.3 \mathrm{THz}$ was observed, which was three to five orders of magnitude larger than the previously reported values. However, their experiment using an individual probe pulse with a $3.6 \mathrm{ps}$ full width at half maximum (FWHM) did not present the timeresolved measurement result. From this literature survey, it is concluded that for the time-resolved transient evolution of water molecular dynamics, only the orientation process excited by low-frequency $\mathrm{THz}$ waves is currently observed. The significant intermolecular modes of liquid water, such as the intermolecular hydrogen bond bending vibration at $\sim 1.8 \mathrm{THz}$ (i.e., $60 \mathrm{~cm}^{-1}$ ) and intermolecular hydrogen bond stretching vibration at $\sim 5.7 \mathrm{THz}$ (i.e., $\left.190 \mathrm{~cm}^{-1}\right)^{15,16,18,19,31,34}$, have not yet been investigated. As the absorption coefficients of water at the frequencies of the intermolecular modes $\left(\alpha=330.18 \mathrm{~cm}^{-1}\right.$ at $1.8 \mathrm{THz}$ and $\alpha=1201.0 \mathrm{~cm}^{-1}$ at $5.7 \mathrm{THz}$ ) are much larger than that at the frequency of the lower-frequency relaxation mode $\left(\alpha=118.01 \mathrm{~cm}^{-1}\right.$ at $0.25 \mathrm{THz})^{35,36}$, the application of the TKE technique in hydrogen bond dynamic research has remained challenging. Furthermore, all of the previous studies adopted cuvettes as liquid containers, which make the extraction of effective information from measurements difficult since the complex molecular interaction force with water and large Kerr response of a cuvette overwhelm the water response ${ }^{18,29,32}$.

Here, an ultrabroadband (centre frequency of $3.9 \mathrm{THz}$ ) and intense (peak electric field strength of $14.9 \mathrm{MV} / \mathrm{cm}$ ) $\mathrm{THz}$ pulse is utilized to excite a gravity-driven, freeflowing water film. The $\mathrm{THz}$ pulse ranges from 1 to $10 \mathrm{THz}$ and covers the two modes of intermolecular motion in the dielectric spectrum of water, which are hydrogen bond bending and stretching vibrations. For the first time, a bipolar TKE signal of liquid water with significant oscillation characteristics is observed. We propose a hydrogen bond harmonic oscillator model to explain the microscopic intermolecular motions. Moreover, we attribute the positive polarity to the intermolecular hydrogen bond stretching vibration and the negative polarity to the hydrogen bond bending vibration. Our theoretical analysis successfully assigns the potential molecular motions and agrees well with the experimental results. Our results provide a more intuitive time-resolved evolution of the polarizability anisotropy induced by the dynamics of the collective intermolecular modes of liquid water on the sub-picosecond scale.

\section{Results}

\section{The Kerr effect in liquid water}

A schematic diagram of the experimental system is shown in Fig. 1a. We use an ultrabroadband $\mathrm{THz}$ pulse with a centre frequency of $3.9 \mathrm{THz}$ to excite the polarizability anisotropy of liquid water (see the "Materials and methods" section and Supplementary Information for details). A gravity-driven flowing water film instead of a traditional cuvette is applied to achieve a high signal-tonoise ratio and improve the accuracy of the experimental data, which is more convincing for analysing the physical characteristics of liquid water. The resulting transient birefringence is monitored by time-delayed optical probe pulses. As shown in Fig. 1b, we obtain a set of bipolar signals with clear oscillatory characteristics under different $\mathrm{THz}$ electric field strengths. We normalize all the curves (see the Supplementary Information for details) and find that all features of the responses scale with the square of the $\mathrm{THz}$ electric field, indicating that the Kerr effect is dominant during this process.

Compared with the negative polarity signal with an obvious relaxation tail extending over a few picoseconds 


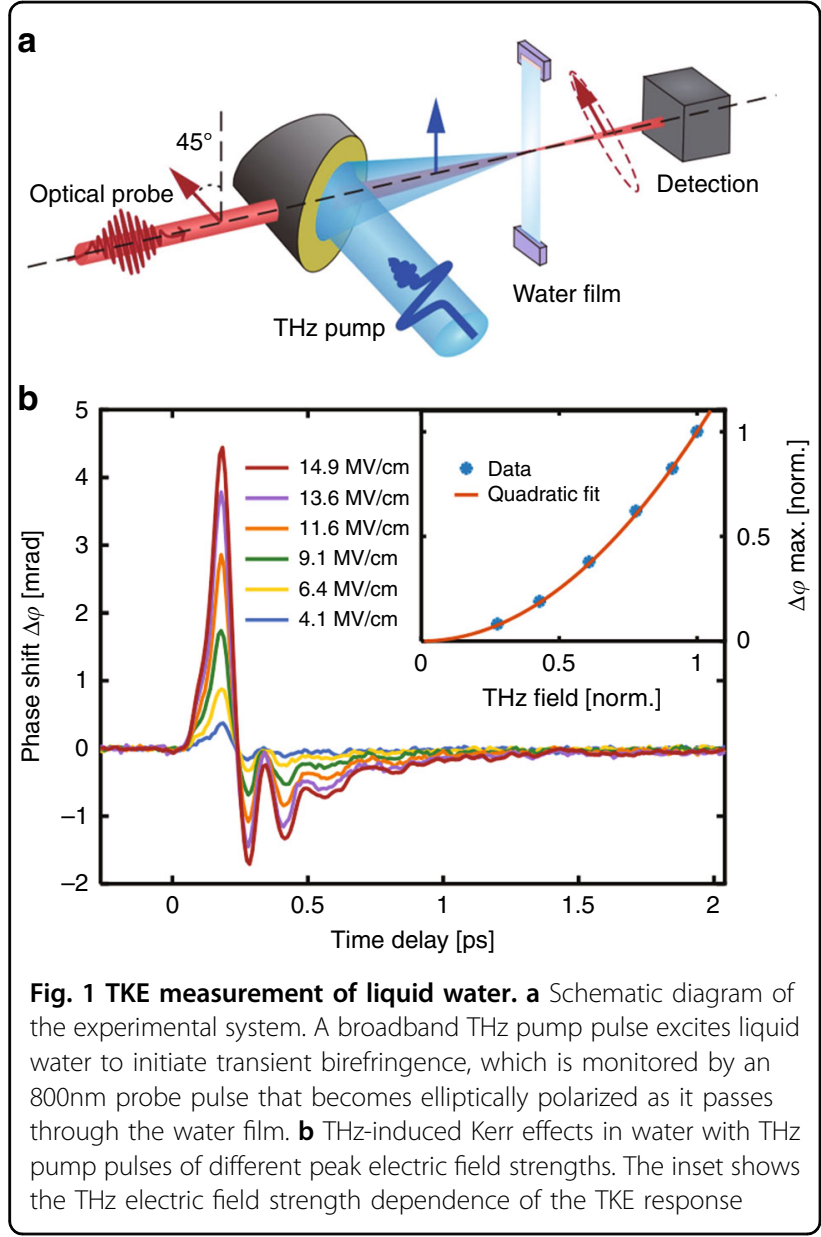

in a previous study ${ }^{32}$, our birefringent signals should have different causes than the molecular orientational relaxation of liquid water. Taking into account the dielectric spectrum of water ${ }^{15,16,18,19,31,34}$, the $\mathrm{THz}$ band in our work mainly covers the two modes of intermolecular hydrogen bond motion, which indicates that the transient birefringence is mainly attributed to the intermolecular structure dynamics of water.

\section{Theoretical model}

The measured phase shift $\Delta \phi$ is positively correlated with the birefringence $\Delta n$, which can be divided into the electronic contribution and molecular contribution. The electronic contribution can be expressed as the multiplication of the square of the electric field with the wavelength and response factor $B_{e}^{32,37}$, i.e., $\Delta n_{e}=\lambda B_{e}|E(z, t)|^{2}$. For the molecular contribution, both the relaxation process and hydrogen bond vibration process contribute to the birefringence signal, i.e., $\Delta n_{m}=$ $\Delta n_{D}+\Delta n_{i}(i=1$ and $i=2$ represent the hydrogen bond bending and stretching vibrations, respectively). $\Delta n_{D}$ is the birefringence caused by the Debye relaxation process,

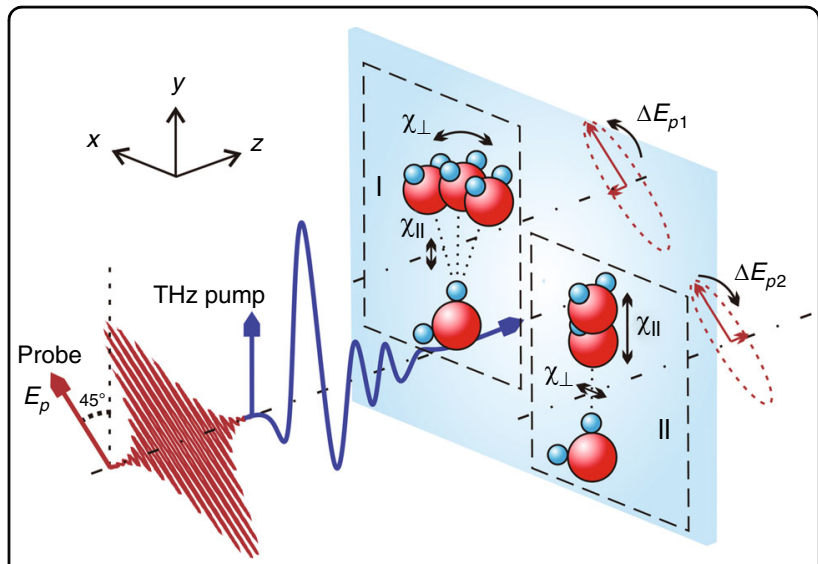

Fig. 2 The effect of hydrogen bond vibrations on the dielectric susceptibility anisotropy. The direction of the hydrogen bond is defined as the parallel direction. (I) In this hydrogen bond unit, if only the bending mode is considered, then the dielectric susceptibility anisotropy is negative $\left(X_{\|}<X_{\perp}\right)$ because the dielectric susceptibility in the vertical direction $\left(X_{\perp}\right)$ is disturbed. (II) In contrast, if only the stretching mode is considered, then the dielectric susceptibility in the parallel direction $\left(X_{\|}\right)$is disturbed. As a result, a linearly polarized probe pulse $\left(E_{p}\right)$ traversing liquid water could acquire ellipticity with opposite handedness because of the two types of molecular motions. A bipolar TKE response combining these two influences is expected

and its intrinsic molecular dynamics have been well explained in previous studies ${ }^{32,38,39}$. However, no study has systematically investigated the contribution to $\Delta n_{i}$ that is related to intermolecular hydrogen bond motions.

Here, the Lorentz dynamic equation is adopted in our model, which is based on the perturbation in the dielectric tensor caused by the intermolecular vibration modes. A spatial rectangular coordinate system is established, as shown in Fig. 2. The beam propagation direction is set as the $z$-axis, and the $\mathrm{THz}$ polarization direction is set as the $y$-axis. We use $Q_{b}$ and $Q_{s}$ to represent the bending and stretching vibration amplitudes of a single hydrogen bond unit, respectively. $Q_{1}=\left\langle Q_{b}\right\rangle$ and $Q_{2}=\left\langle Q_{s}\right\rangle$ represent the average amplitude tensors of the hydrogen bond bending and stretching modes per unit volume, respectively. Without the THz pump electric field, the water molecular motions are in an isotropic thermodynamic equilibrium state, and the amplitudes of the hydrogen bond vibration in all directions are equal. When a $\mathrm{THz}$ electric field is applied in the y direction, the instantaneous rotation of water molecules provides an additional driving potential for the intermolecular hydrogen bond bending and stretching vibrations ${ }^{14,32}$.

According to the Taylor series, in one hydrogen bond unit, the perturbations of the dielectric susceptibilities parallel and perpendicular to the hydrogen bond direction caused by the intermolecular modes are expressed as follows (see the "Materials and methods" section for 
details):

$$
X_{\|}=X_{0}+\frac{\partial X_{\|}}{\partial Q_{b}} Q_{b}+\frac{\partial X_{\|}}{\partial Q_{s}} Q_{s}, X_{\perp}=X_{0}+\frac{\partial X_{\perp}}{\partial Q_{b}} Q_{b}+\frac{\partial X_{\perp}}{\partial Q_{s}} Q_{s}
$$

For the bending mode, the hydrogen bond oscillator mainly perturbs the dielectric susceptibility component vertical to the hydrogen bond direction, which is represented by phenomenon (I) in Fig. 2, i.e., $\frac{\partial X_{\|}}{\partial Q_{b}} Q_{b} \ll \frac{\partial X_{\perp}}{\partial Q_{b}} Q_{b}$, where $\frac{\partial X_{\|}}{\partial Q_{b}} Q_{b}$ can be ignored. Similarly, the perturbation of the dielectric susceptibility caused by the stretching vibration is mainly in the parallel direction with respect to the hydrogen bond, which is depicted by phenomenon (II) in Fig. 2. Therefore, the perturbation of the dielectric susceptibility vertical to the hydrogen bond direction mainly originates from the bending vibration, i.e., $X_{\perp} \approx X_{0}+\frac{\partial X_{\perp}}{\partial Q_{b}} Q_{b}$, and that parallel to the hydrogen bond direction mainly comes from the stretching vibration, i.e., $X_{\|} \approx X_{0}+\frac{\partial X_{\|}}{\partial Q_{s}} Q_{s}$.

The macroscopic expression of the dielectric tensor is the average value of all the vectors of the microscopic units per unit volume. The driving $\mathrm{THz}$ electric field (y-polarized) will cause instantaneous rotation of the water molecule and provide a driving potential in the $y$ direction. Due to the directional relationship between the hydrogen bond and the permanent dipole moment of the water molecule, the y component of the hydrogen bond will obtain more coupling energy ${ }^{24}$. The anisotropic perturbation caused by the intermolecular vibration modes excited by the y-polarized $\mathrm{THz}$ electric field is defined as $q_{i}=Q_{i} y-\frac{1}{2}\left(Q_{i} x+Q_{i} z\right)>0\left(q_{1}\right.$ and $q_{2}$ represent the anisotropic perturbations caused by hydrogen bond bending and stretching vibrations, respectively). The resulting polarizability anisotropy can be expressed by the refractive index associated with the dielectric susceptibility $X$ (see the "Materials and methods" section for details) ${ }^{40}$ :

$$
\sum_{i=1,2} \Delta n_{i}=\frac{1}{2 \varepsilon_{0} n_{0}}\left[\varepsilon_{y}-\frac{1}{2}\left(\varepsilon_{x}+\varepsilon_{z}\right)\right]=\frac{1}{2 n_{0}}\left(\frac{\partial X_{\|}}{\partial Q_{s}} q_{2}-\frac{\partial X_{\perp}}{\partial Q_{b}} q_{1}\right)
$$

where $\varepsilon_{0}$ is the vacuum dielectric constant and $n_{0}$ is the refractive index of liquid water. $q_{i}$ mentioned above is positive. These two intermolecular motions result in opposite birefringence contributions to the total refractive index, i.e., $\Delta n_{1}<0, \Delta n_{2}>0$.

In addition, $q_{i}$ satisfies the Lorentz dynamic model, which describes the motion of the damped harmonic oscillator $^{41,42}$ (see the "Materials and methods" section for details):

$$
\frac{\partial^{2} q_{i}(t)}{\partial t^{2}}+\gamma_{i} \frac{\partial q_{i}(t)}{\partial t}+\omega_{i}^{2} q_{i}(t)=a_{i} E^{2}(t) \quad(i=1,2)
$$

where $\gamma_{i}, \omega_{i}$ represent the damping coefficient and inherent frequency, respectively, with values ${ }^{15}$ of $\gamma_{1}=$ $115 \mathrm{~cm}^{-1}, \quad \gamma_{2}=165 \mathrm{~cm}^{-1}, \quad \omega_{1}=60 \mathrm{~cm}^{-1}$ and $\omega_{2}=$ $190 \mathrm{~cm}^{-1}$. Here, $a_{i}=\beta_{i} \frac{\mu_{0}^{2}}{3 \sqrt{2} k_{B} T m}$ represents the coupling factor between the square of the THz pump electric field and the driving term in Eq. (3). A detailed description of $a_{i}$ can be found in the "Materials and methods" section.

\section{Simulation and analysis}

We use the above model to decompose and simulate the experimental data in the time domain, as shown in Fig. 3a-c. The data can also be decomposed into (i) positive responses with electronic (blue line) and hydrogen bond stretching (purple line) contributions and (ii) negative responses with Debye relaxation (red line) and hydrogen bond bending (yellow line) contributions. To validate our proposed model, we change the frequency range of the $\mathrm{THz}$ pump pulse by using different low-pass filters (LPFs). As shown in Fig. 3a-c, the simulation results based on our proposed model are in good agreement with the measured data. As the cut-off frequency decreases, the positive polarity response is more significantly reduced than the negative polarity response, indicating that the positive response is mainly derived from higher-frequency molecular motion. In addition, the weak electronic contribution of water can be attributed to the very small hyperpolarizabilities in water ${ }^{32,43}$.

We find that the molecular contribution exhibits a strong frequency dependence due to the participation of pertinent molecular motion mechanisms. Here, we use an ultrabroadband $\mathrm{THz}$ pulse to excite liquid water, and the induced transient anisotropy is dominated by the intermolecular hydrogen bond stretching and bending vibrations. As the frequency decreases, the ratio of the anisotropic contribution caused by the intermolecular modes to the electronic contribution increases. This phenomenon is reasonable for intermolecular modes with characteristic frequencies less than $6 \mathrm{THz}$. Moreover, the latest experiment on $\mathrm{THz}$-driven liquid water shows that with a lower centre frequency excitation $(\sim 0.25 \mathrm{THz})$, the orientation process guides the anisotropic distribution, showing a negative TKE response ${ }^{32}$. However, Kampfrath et al. observed a bipolar Kerr effect of liquid water with an $\sim 1 \mathrm{THz}$ centre frequency for excitation ${ }^{31}$, which suggested that the molecular response mechanism depends critically on the driving frequency. For a relatively high $\mathrm{THz}$ frequency excitation (such as $\sim 3.9 \mathrm{THz}$ centre 

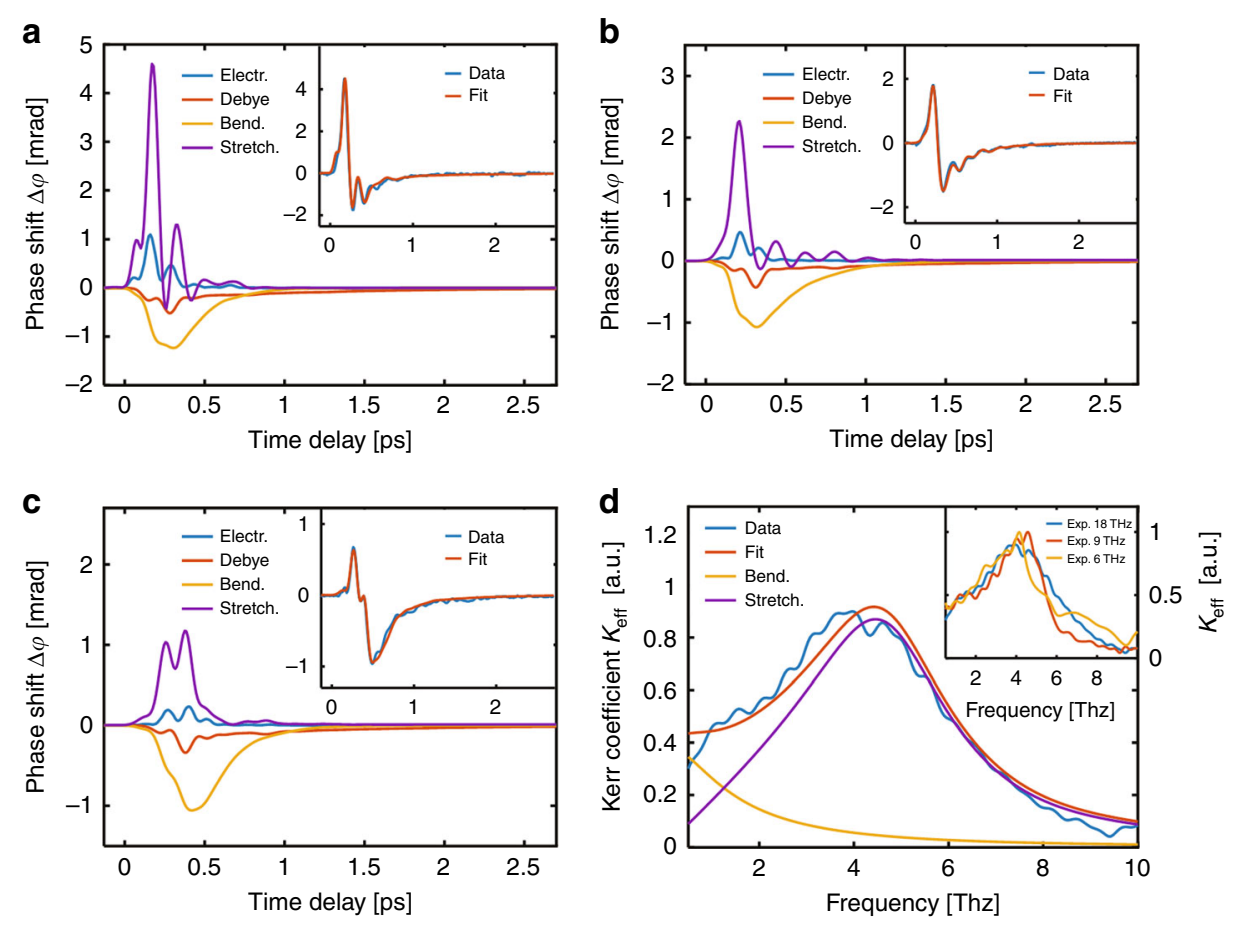

Fig. 3 Simulation results of measurement data. Theoretical simulation of the TKE response in the time domain for the electronic, Debye relaxation, hydrogen bond stretching vibration and hydrogen bond bending vibration contributions under different pump electric fields with cut-off frequencies of $\mathbf{a} 18 \mathrm{THz}, \mathbf{b} 9 \mathrm{THz}$ and $\mathbf{c} 6 \mathrm{THz}$. The corresponding inset shows a comparison between the sum of all the contributions and the measured data. $\mathbf{d}$ Theoretical frequency-domain Kerr coefficient $K_{\text {eff }}$ under hydrogen bond bending (yellow line) and stretching (purple line) modes. The sum of these two theoretical Kerr coefficients (red line) closely matches the data extracted from the experimental results with a cut-off frequency of $18 \mathrm{THz}$ (blue line). The inset shows the measured Kerr coefficient $K_{\text {eff }}$ extracted from the birefringent signals under different electric fields with cutoff frequencies of 18,9 and $6 \mathrm{THz}$

frequency), the molecular orientation process loses its dominant position, and intermolecular modes with obvious damping characteristics dominate. It can be predicted that the librational modes need to be considered when using a pulse with a higher $\mathrm{THz}$ frequency excitation (such as frequencies above $12 \mathrm{THz})^{16}$.

We mainly attribute the bipolar TKE signal to the contributions of the two distinct modes of hydrogen bond vibrations rather than to the electronic or Debye contribution. Here, we perform a Fourier transform of the homogeneous solutions of the Lorentz time-domain differential equations for hydrogen bond bending and stretching modes in our model and obtain the theoretical frequency-domain Kerr coefficient in these two intermolecular modes, as shown by the yellow and purple lines in Fig. 3d. Moreover, we extract the frequency-domain Kerr coefficient $K_{\text {eff }}\left(\mathcal{F}(\Delta n(t)) / \mathcal{F}\left(E^{2}(t)\right)\right)$ from our experimental results for the three input $\mathrm{THz}$ electric fields over the range of $0.5-10 \mathrm{THz}$, and this coefficient has a high signal-to-noise ratio and mainly covers the intermolecular modes. A significant peak appears near $4.5 \mathrm{THz}$ and corresponds to the contribution of the hydrogen bond stretching vibration. Since the hydrogen bond bending vibration is approximately a critically damped harmonic oscillation, no apparent peak position is observed. Similarly, for the TKE time-domain responses of liquid water, the apparent oscillation features are primarily due to the underdamped harmonic oscillation of stretching. We find that the measured Kerr coefficient (blue line) can be approximated by the sum of the two intermolecular mode contributions (red line). The Kerr coefficient response induced by intermolecular hydrogen bond vibrations can be simply approximated by the following formula:

$$
K_{\text {eff }}(\omega)=\sum_{i=1,2} \frac{\left|k_{i}\right|}{\omega_{i}^{2}-\omega^{2}+i \gamma_{i} \omega}
$$

where the real part of $K_{\text {eff }}$ is the Kerr coefficient. $k_{i}$ is the scaling constant of the $\mathrm{THz}$ Kerr effect ${ }^{44}$, i.e., $k_{1}=$ $\frac{a_{1}}{\lambda}\left(\frac{\partial X_{\|}}{\partial Q_{b}}-\frac{\partial X_{\perp}}{\partial Q_{b}}\right)$ and $k_{2}=\frac{a_{2}}{\lambda}\left(\frac{\partial X_{\|}}{\partial Q_{s}}-\frac{\partial X_{\perp}}{\partial Q_{s}}\right) \cdot k_{1}$ and $k_{2}$ calculated from the experimental data are $k_{1} \approx-7 \times 10^{9} \mathrm{C} /(\mathrm{V} \cdot \mathrm{kg} \cdot \mathrm{m})$ and $k_{2} \approx 6.5 \times 10^{10} \mathrm{C} /(\mathrm{V} \cdot \mathrm{kg} \cdot \mathrm{m})$, respectively. 

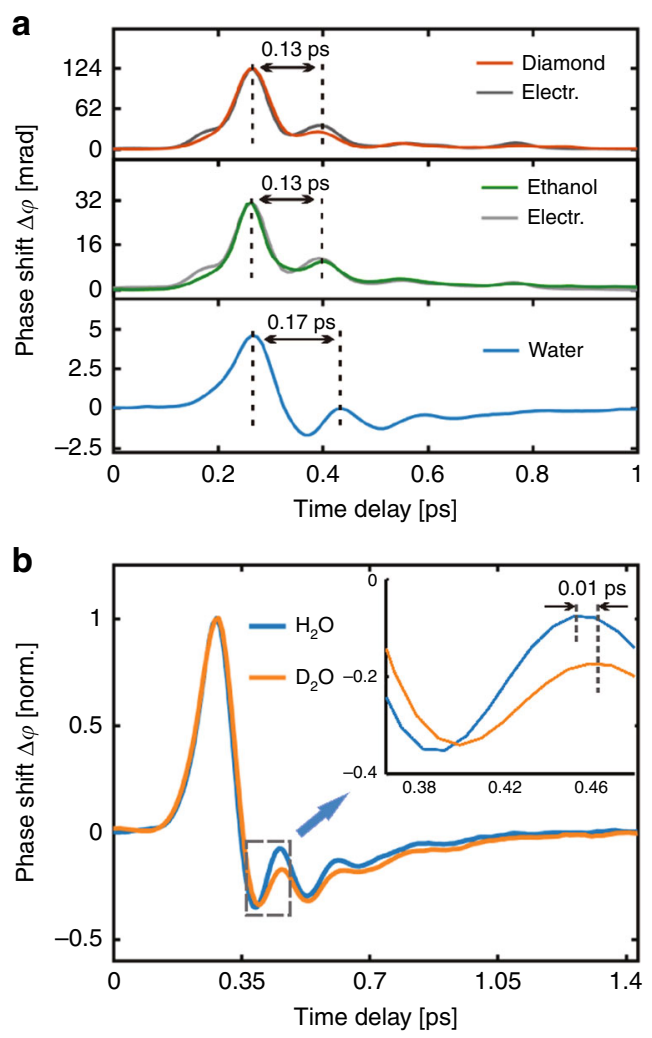

Fig. 4 TKE responses of different materials. a The TKE responses of diamond (top-red) and ethanol (middle-green) exhibit similar doublepeak structures with a time interval of $0.13 \mathrm{ps}$. The simulated electronic responses of diamond and ethanol are shown as dark grey and light grey lines, respectively. In comparison, the TKE response of liquid water (bottom) comprises a broadened double-peak structure with a time interval of $0.17 \mathrm{ps}$ and an obvious negative component. $\mathbf{b}$ The

TKE responses of liquid water and heavy water are demonstrated for comparison

To further confirm that our birefringent signal of liquid water mainly comes from unique hydrogen bond motions rather than the electronic contribution, we carry out comparative measurements of ethanol, diamond and water under a pump electric field with a cut-off frequency of $18 \mathrm{THz}$. The TKE signals of diamond and ethanol exhibit a double-peak structure, which is a typical Kerrtype response curve, as shown in the top and middle panels of Fig. 4a. In comparison, the TKE signal of liquid water has not only a distinct negative component but also a broadened double-peak structure, as shown in the bottom panel of Fig. 4a. Theoretically, diamond as an ultrafast pulse switching, its TKE response curve will fully follow the square of the $\mathrm{THz}$ field ${ }^{45,46}$. Here, we simulate the electronic response by considering the influence factors of sampling error and the $\mathrm{THz}$ wave transmission process in the sample (such as the phase shift of a $\mathrm{THz}$ wave during its transmission in the sample, the attenuation of $\mathrm{THz}$ intensity in the sample, and the dispersion between the $\mathrm{THz}$ wave and the probe light in the sample) ${ }^{35,46,47}$. We obtain the expected fitting curve of the diamond's electronic response, which is in good agreement with the experimental data. Similarly, the simulated electronic response of ethanol agrees well with the experimental data, revealing that the TKE signal of ethanol is mainly dominated by the electronic response. It is worth noting that Kampfrath et al. also observed that the TKE signal of ethanol is dominated by the electronic response under a pump $\mathrm{THz}$ field with $\sim 1 \mathrm{THz}$ centre frequency ${ }^{27}$. However, Zalden et al. used THz pulses with $\sim 0.25 \mathrm{THz}$ centre frequency to excite ethanol and obtained a relatively high molecular response ${ }^{32}$, revealing that the molecular response of ethanol depends heavily on the driving frequency ${ }^{48}$. Compared with diamond and ethanol, water has a much weaker electronic response (as shown in Fig. 3a), which is due to the small effective medium length $(\sim 25-\mu \mathrm{m}$ thick water can absorb $90 \%$ of $\mathrm{THz}$ energy in our experiment) and the small electronic response factor $B_{e}\left(B_{e}:<0.003 \times 10^{-14} \mathrm{~m} / \mathrm{V}^{2}\right.$ for liquid water, $\sim 0.00996 \times 10^{-14} \mathrm{~m} / \mathrm{V}^{2}$ for diamond, and $\sim 0.0093 \times 10^{-14} \mathrm{~m} / \mathrm{V}^{2}$ for ethanol) $)^{32,43,46}$. In addition, the simulated electronic response of water is closer to the shape of the $\mathrm{THz}$ intensity curve, mainly because the small effective medium length limits the distortion of the electronic response introduced by the $\mathrm{THz}$ wave transmission process in the sample. Moreover, the $\mathrm{THz}$ intensity curve with $\sim 3.9 \mathrm{THz}$ centre frequency has the characteristic of a double-peak time interval of $0.13 \mathrm{ps}$, and this characteristic is also displayed in the electronictype TKE response curves of diamond and ethanol. In comparison, the TKE signal of water exhibits a broadened double-peak time interval of $0.17 \mathrm{ps}$, which represents the damped oscillation period corresponding to the hydrogen bond stretching vibration with a characteristic frequency of $5.7 \mathrm{THz}$. In summary, we believe that the unique TKE response of water with the broadened double-peak characteristic mainly originates from the contribution of intermolecular modes rather than the electronic response.

To verify the applicability of our model, we measure the TKE response of heavy water and show it in Fig. 4b. Compared with that of water, the characteristic frequency of the hydrogen bond stretching vibration of heavy water exhibits a slight red shift ${ }^{15}$, which results in a slight broadening of the double-peak time interval of the TKE response. In addition, the relatively large damping coefficient of heavy water in the stretching mode corresponds to the faster energy decay process of the harmonic oscillator, resulting in a reduction in the second peak of the TKE response compared to that of water. Moreover, our model is used in frequency-dependent simulations of heavy water and matches well with experimental results (see section SIII in the Supplementary Information for details). 


\section{Discussion}

We use a $\mathrm{THz}$ electric field to resonantly excite the intermolecular modes of liquid water. We believe that the transient rotation of a molecule produces an induced dipole moment, which immediately transfers the momentum driven by the $\mathrm{THz}$ field to the restricted translational motion of adjacent water molecules. This translational motion can be assigned to a bending mode and a stretching mode, which can lead to the components of polarizability anisotropy perpendicular and parallel to the hydrogen bonds, respectively, thus resulting in bidirectional performance. This assignment is also confirmed by Raman spectroscopy ${ }^{49}$.

In recent studies of $\mathrm{THz}$-excited reorientational diffusive motions in liquid water, the authors explained the negative contribution origins from molecular orientation or intermolecular motion due to a low-frequency $\mathrm{THz}$ pulse $(<1 \mathrm{THz} \text { centre frequency })^{31,32}$. However, they described the measured positive component simply as a fast relaxation process with exponential decay ${ }^{31}$. In our experiment, the measured TKE response shows a distinctly damped oscillation process with a $170 \mathrm{fs}$ doublepeak time interval, indicating that this phenomenon cannot be explained by a fast relaxation process. Moreover, the sub-picosecond time scale of $170 \mathrm{fs}$ is consistent with the characteristic frequency of the hydrogen bond stretching vibration, revealing that the positive Kerr effect is mainly derived from the stretching vibration rather than a fast relaxation process. Furthermore, compared with the negative response with a relaxation tail extending over a few picoseconds reported in the literature ${ }^{31,32}$, our negative response exhibits a feature on the sub-picosecond scale. Through our theoretical simulation, we conclude that this feature is mainly derived from a damped oscillation process of the bending mode rather than a molecular orientation process.

We infer that our method has the potential to detect the physical mechanism of damped harmonic oscillator processes at higher frequencies ${ }^{16}$, such as 420 and $620 \mathrm{~cm}^{-1}$. However, due to the response function of our instrument, consideration of frequencies higher than $400 \mathrm{~cm}^{-1}$ is not necessary. In addition, according to the multicomponent schematic formulation of the main mode-coupling theory $(\mathrm{SMC})^{22}$, high-frequency vibrational contributions $\left(>400 \mathrm{~cm}^{-1}\right)$ do not affect the response function in the lower-frequency range that our work investigated.

In conclusion, we have demonstrated that the lowfrequency molecular motion patterns of liquid water molecules are excited by an intense and ultrabroadband $\mathrm{THz}$ electric field. The TKE response is assigned to the superposition of four components, among which the bidirectional contributions of bending and stretching modes play dominant roles. We proposed a hydrogen bond oscillator model based on the Lorentz dynamic equation to describe the dynamics of the intermolecular modes of liquid water and successfully reproduced the measured TKE responses. A Kerr coefficient response equation was further established to study the influence of the underlying parameters. We believe that our method can also be applied to detect the physical mechanisms of the gas phase of water and crystalline and amorphous ices, as well as the complex interaction of reagents with solvent water molecules.

\section{Materials and methods \\ Experimental setup}

The experimental setup is schematically depicted in Fig. $\mathrm{S} 1$ in the Supplementary Information. The laser produced by a $5 \mathrm{~mJ}$ Ti:sapphire laser system (Spitfire, Spectra Physics) with a central wavelength of $800 \mathrm{~nm}$ and a repetition rate of $1 \mathrm{kHz}$ is separated into two beams by a beam splitter with a 7:3 (R:T) split ratio. One of the $3.5 \mathrm{~mJ}$ beams is used to pump a commercial optical parametric amplifier (TOPAS, Spectra Physics), which delivers an optical laser beam with a wavelength of $1550 \mathrm{~nm}$. The other beam is used as a probe laser with a wavelength of $800 \mathrm{~nm}$. An organic 4-N,N-dimethylamino-4'-N'-methylstilbazoliumtosylate (DAST) crystal (Swiss Terahertz LLC) is pumped by the optical beam with a $1550 \mathrm{~nm}$ wavelength, and a $\mathrm{THz}$ wave is collinearly generated. Residual NIR is filtered out using three low-pass filters (QMC Instruments Ltd) with cut-off frequencies of $18 \mathrm{THz}$. The THz beam is expanded and then focused by three off-axis parabolic mirrors. The energy of the $\mathrm{THz}$ wave is controlled by two THz polarizers (POL-HDPECA50-OD63-T8, Tydex). Two aluminium wires with diameters of $100 \mu \mathrm{m}$ are separated by $\sim 5 \mathrm{~mm}$ to form a stable free-flowing water film. The thickness of the water film is $90 \pm 4 \mu \mathrm{m}$ and can be adjusted by controlling the water flow rate. Our stable free-flowing water film can effectively eliminate the heat accumulation between two adjacent $\mathrm{THz}$ pump pulses, and the contribution of heating effects to the TKE signal in the experiment is negligible (see section SVI in the Supplementary Information for details). An optical second-harmonic intensity autocorrelator is used to measure and calibrate the thickness $^{50}$.

The linearly polarized $\mathrm{THz}$ pulse is focused on liquid water. The polarization of the probe beam with an $800 \mathrm{~nm}$ wavelength is tilted by $45^{\circ}$ relative to the $\mathrm{THz}$ electric field polarization. The time trajectory is recorded by scanning the delay (Here, an electric stage type M-ILS100PP purchased from Newport Corporation is used.) between the pump and probe beams and reading the modulation caused by the $\mathrm{THz}$ pump pulse detected by a balanced photodiode (PDB210A/M, Thorlabs) using a lock-in amplifier (Model SR830 DSP, Stanford Research Systems). The system is purged with dry nitrogen gas to 
eliminate the absorption of water vapour. However, nitrogen gas has a small TKE response under our experimental conditions. Therefore, the background response of nitrogen gas has been subtracted from the data presented in the article (see section SV in the Supplementary Information for details). The resulting birefringence $\Delta n(z, t)$ is detected based on the phase shift $\Delta \phi(t)$ at position $z$ along the propagation direction ${ }^{51,52}$ via:

$$
\Delta \phi(t)=\frac{2 \pi}{\lambda} \int_{0}^{l} \Delta n(z, t) d z
$$

\section{Sample source}

Ultra-pure water $(\sigma<0.1 \mu \mathrm{S} / \mathrm{cm})$ is obtained from a labbased purification system. Heavy water with $99 \%$ purity is commercially obtained from CIL (USA). Ethanol with $>99.8 \%$ purity is commercially obtained from HUSHI $(\mathrm{CHN})$. In our work, both water and ethanol have a thickness of $90 \mu \mathrm{m}$ by using a stable free-flowing liquid film device. The diamond substrate is a single crystal and has a thickness of $0.3 \mathrm{~mm}$. It is purchased from Element Six (UK). During all measurements, the surrounding environment is held at a constant temperature of $22 \pm$ $1{ }^{\circ} \mathrm{C}$.

\section{Model details}

The perturbation of the dielectric susceptibility caused by molecular vibrations can be expanded into the following Taylor series according to the components of the amplitude:

$$
\chi=\chi_{0}+\sum_{i} \frac{\partial \chi}{\partial Q_{i}} Q_{i}+\frac{1}{2} \sum_{i, j} \frac{\partial^{2} \chi}{\partial Q_{1} \partial Q_{2}} Q_{i} Q_{j}+\ldots \ldots
$$

Then, the dielectric function can be written as $\varepsilon=$ $\varepsilon_{0}\left(\varepsilon_{r}+\chi\right)$, where $\varepsilon_{r}$ is the relative dielectric constant of the $800 \mathrm{~nm}$ probe pulse without $\mathrm{THz}$ wave pumping. Through experimental data, we find that the signal response has good linearity with the square of the electric field, which proves that the polarization anisotropy of water molecules is mainly derived from second-order polarization and has no higher-order terms. Therefore, the above formula can be simplified as:

$$
X=\chi_{0}+\sum_{i} \frac{\partial X}{\partial Q_{i}} Q_{i}
$$

In this work, the intermolecular hydrogen bond motions of liquid water have two significant oscillator vibration modes in the $\mathrm{THz}$ range: bending vibration and stretching vibration. The dielectric tensor after the spindle transformation is $\varepsilon=\left[\begin{array}{lll}\varepsilon_{x} & \varepsilon_{y} & \varepsilon_{z}\end{array}\right]^{T}$. According to Eq. (7), the dielectric tensor can be expressed as:

$$
\left[\begin{array}{c}
\varepsilon_{x} \\
\varepsilon_{y} \\
\varepsilon_{z}
\end{array}\right]=\varepsilon_{0}\left[1+\chi_{0}\right] I+\varepsilon_{0}\left\{\left[\begin{array}{l}
\frac{\partial X_{\|}}{\partial Q_{b}}, \frac{\partial X_{\perp}}{\partial Q_{1}}, \frac{\partial X_{\perp}}{\partial Q_{b}} \\
\frac{\partial X_{\perp}}{\partial Q_{b}}, \frac{\partial X_{1}}{\partial Q_{b}}, \frac{\partial X_{\perp}}{\partial Q_{b}} \\
\frac{\partial X_{\perp}}{\partial Q_{b}}, \frac{\partial X_{\perp}}{\partial Q_{b}}, \frac{\partial X_{\|}}{\partial Q_{b}}
\end{array}\right]\left[\begin{array}{l}
Q_{1} x \\
Q_{1} y \\
Q_{1} z
\end{array}\right]+\left[\begin{array}{l}
\frac{\partial X_{\|}}{\partial Q_{s}}, \frac{\partial X_{\perp}}{\partial Q_{s}}, \frac{\partial X_{\perp}}{\partial Q_{s}} \\
\frac{\partial X_{\perp}}{\partial Q_{s}}, \frac{\partial X_{\|}}{\partial Q_{s}}, \frac{\partial X_{\perp}}{\partial Q_{s}} \\
\frac{\partial X_{\perp}}{\partial Q_{s}}, \frac{\partial X_{\perp}}{\partial Q_{s}}, \frac{\partial X_{\|}}{\partial Q_{s}}
\end{array}\right]\left[\begin{array}{c}
Q_{2} x \\
Q_{2} y \\
Q_{2} z
\end{array}\right]\right\}
$$

Therefore, through the deduction of the above formula, the polarization anisotropy $\Delta n_{i}$ caused by $\mathrm{THz}$ wave driving can be expressed as Eq. (2).

For an oscillator with amplitude $Q$, mass $m$, and inherent frequency $\omega$, the molecular harmonic oscillator equation can be expressed as ${ }^{41,42}$ :

$$
\frac{\partial^{2} Q}{\partial t^{2}}+\gamma \frac{\partial Q}{\partial t}+\omega^{2} Q=\frac{\partial W}{m \partial Q}
$$

where $W$ is the potential energy of the oscillator driven by the electric field. In this model, the potential energy of the $\mathrm{THz}$ electric field for driving the instantaneous rotation of water molecules is $E \cdot \mu_{\text {ind }}$. The induced dipole moment caused by the instantaneous rotation of the permanent dipole moment of a water molecule can be expressed as $\mu_{\text {ind }}=\Delta \alpha E=\mu_{0}\langle\cos \theta\rangle$, where $\Delta \alpha=\alpha_{\|}-\alpha_{\perp}$ means that the polarizability of the water molecule parallel to the direction of the permanent dipole moment is smaller than the polarizability perpendicular to the permanent dipole moment. We can establish the relationship between $\langle\cos \theta\rangle$ and the $\mathrm{THz}$ electric field $E$ based on the theory of Kalmykov and Coffey ${ }^{38,39}$ :

$$
\langle\cos \theta\rangle \approx \frac{\left|\mu_{0}\right|}{3 \sqrt{2} k_{B} T} E(t) * R(t)
$$

Due to the instantaneous rotation of water molecules during synergy with the hydrogen bond oscillator, the coupling potential energy obtained by the hydrogen bond oscillator is positively correlated with its amplitude. Therefore, the potential energy can be given as:

$$
W(t) \approx \beta Q \frac{\mu_{0}^{2}}{3 \sqrt{2} k_{B} T} R(t) * E^{2}(t)
$$

where $\beta\left(\mathrm{m}^{-1}\right)$ is the coupling coefficient and $R(t)$ represents the fast Debye relaxation process of water molecules, which has a time constant of $\sim 0.1 \mathrm{ps}$; thus, the right-hand side of the harmonic oscillator equation can be simply given as $\frac{\partial W}{m \partial Q} \approx \beta \frac{\mu_{0}^{2}}{3 \sqrt{2} k_{B} T m} E^{2}(t)$. Since $q_{i}$ is linearly related to $Q, q_{i}$ also satisfies the Lorentz oscillation process described by Eq. (3), and in this case, the driving term on the right-hand side of Eq. (3) can be given as $a_{i} E^{2}(t)$, where $a_{i}=\beta_{i} \frac{\mu_{0}^{2}}{3 \sqrt{2} k_{B} T m}$. 


\section{Acknowledgements}

This work was supported by the Beijing Natural Science Foundation (Grant No. JQ18015), the National Natural Science Foundation of China (Grant Nos. 61935001 and 61905271), the Guangdong Basic and Applied Basic Research Foundation (Grant No. 2020A1515011083), and the Beijing Advanced Innovation Center for Imaging Theory and Technology.

\section{Author details}

'Beijing Key Laboratory for Precision Optoelectronic Measurement Instrument and Technology, School of Optics and Photonics, Beijing Institute of Technology, Beijing 100081, China. ${ }^{2}$ Beijing Advanced Innovation Center for Imaging Technology and Key Laboratory of Terahertz Optoelectronics (MoE), Department of Physics, Capital Normal University, Beijing 100048, China. ${ }^{3}$ Shenzhen Institutes of Advanced Technology, Chinese Academy of Sciences, Shenzhen 518055, China. ${ }^{4}$ The Institute of Optics, University of Rochester, Rochester, NY 14627, USA

\section{Author contributions}

H.Z. and Y.T. built the setup and carried out measurements, and experimental data were simulated and analysed by Y.T., H.Z., L.Z., Y.Z. and X.Z. The project was conceived and designed by L.Z., Y.Z., H.Z. and Y.T. The theoretical feasibility of the experiment and the reliability of the data analysis were verified by R.Z., C.Z., M.S. and X.Z. All the authors contributed to the writing of this paper.

\section{Conflict of interest}

The authors declare that they have no conflict of interest.

Supplementary information is available for this paper at https://doi.org/ 10.1038/s41377-020-00370-z.

Received: 23 September 2019 Revised: 4 July 2020 Accepted: 13 July 2020 Published online: 04 August 2020

\section{References}

1. Cisneros, G. A. et al. Modeling molecular interactions in water: from pairwise to many-body potential energy functions. Chem. Rev. 116, 7501-7528 (2016).

2. Bellissent-Funel, M. C. et al. Water determines the structure and dynamics of proteins. Chem. Rev. 116, 7673-7697 (2016).

3. Omta, A. W. et al. Negligible effect of ions on the hydrogen-bond structure in liquid water. Science 301, 347-349 (2003).

4. Fecko, C. J. et al. Ultrafast hydrogen-bond dynamics in the infrared spectroscopy of water. Science 301, 1698-1702 (2003).

5. Smith, J. D. et al. Energetics of hydrogen bond network rearrangements in liquid water. Science 306, 851-853 (2004).

6. Stiopkin, I. V. et al. Hydrogen bonding at the water surface revealed by isotopic dilution spectroscopy. Nature 474, 192-195 (2011).

7. Richardson, J. O. et al. Concerted hydrogen-bond breaking by quantum tunneling in the water hexamer prism. Science 351, 1310-1313 (2016).

8. Stokely, K. et al. Effect of hydrogen bond cooperativity on the behavior of water. Proc. Natl Acad. Sci. USA 107, 1301-1306 (2010).

9. Sharma, M., Resta, R. \& Car, R. Intermolecular dynamical charge fluctuations in water: a signature of the H-bond network. Phys. Rev. Lett. 95, 187401 (2005).

10. Heyden, M. \& Tobias, D. J. Spatial dependence of protein-water collective hydrogen-bond dynamics. Phys. Rev. Lett. 111, 218101 (2013).

11. Bakker, H. J. \& Skinner, J. L. Vibrational spectroscopy as a probe of structure and dynamics in liquid water. Chem. Rev. 110, 1498-1517 (2010).

12. Perakis, F. et al. Vibrational spectroscopy and dynamics of water. Chem. Rev. 116, 7590-7607 (2016).

13. Vij, J. K., Simpson, D. R. J. \& Panarina, O. E. Far infrared spectroscopy of water at different temperatures: $\mathrm{GHz}$ to THz dielectric spectroscopy of water. J. Mol. Liq. 112, 125-135 (2004).

14. Torii, $H$. Intermolecular electron density modulations in water and their effects on the far-infrared spectral profiles at 6 THz. J. Phys. Chem. B 115, 6636-6643 (2011).

15. Mizoguchi, K., Hori, Y. \& Tominaga, Y. Study on dynamical structure in water and heavy water by low-frequency Raman spectroscopy. J. Chem. Phys. 97, 1961-1968 (1992).
16. Fukasawa, T. et al. Relation between dielectric and low-frequency Raman spectra of hydrogen-bond liquids. Phys. Rev. Lett. 95, 197802 (2005).

17. Rønne, C. \& Keiding, S. R. Low frequency spectroscopy of liquid water using THz-time domain spectroscopy. J. Mol. Liq. 101, 199-218 (2002).

18. Penkov, N. et al. Terahertz spectroscopy applied for investigation of water structure. J. Phys. Chem. B 119, 12664-12670 (2015).

19. Savolainen, J., Ahmed, S. \& Hamm, P. Two-dimensional Raman-terahertz spectroscopy of water. Proc. Natl Acad. Sci. USA 110, 20402-20407 (2013).

20. Torre, R., Bartolini, P. \& Righini, R. Structural relaxation in supercooled water by time-resolved spectroscopy. Nature 428, 296-299 (2004).

21. Turton, D. A. \& Wynne, K. Structural relaxation in the hydrogen-bonding liquids $\mathrm{N}$-methylacetamide and water studied by optical Kerr effect spectroscopy. J. Chem. Phys. 128, 154516 (2008).

22. Taschin, A. et al. Evidence of two distinct local structures of water from ambient to supercooled conditions. Nat. Commun. 4, 2401 (2013).

23. Winkler, K., Lindner, J. \& Vöhringer, P. Low-frequency depolarized Ramanspectral density of liquid water from femtosecond optical Kerr-effect measurements: lineshape analysis of restricted translational modes. Phys. Chem. Chem. Phys. 4, 2144-2155 (2002).

24. Sajadi, M., Wolf, M. \& Kampfrath, T. Transient birefringence of liquids induced by terahertz electric-field torque on permanent molecular dipoles. Nat. Commun. 8, 14963 (2017).

25. Freysz, E. \& Degert, J. Nonlinear optics: terahertz Kerr effect. Nat. Photon. 4, 131-132 (2010).

26. Finneran, I. A. et al. Coherent two-dimensional terahertz-terahertz-Raman spectroscopy. Proc. Natl Acad. Sci. USA 113, 6857-6861 (2016).

27. Kampfrath, T. et al. The nature of the dielectric response of methanol revealed by the terahertz Kerr effect. J. Phys. Chem. Lett. 9, 1279-1283 (2018).

28. Hoffmann, M. C. et al. Terahertz kerr effect. Appl. Phys. Lett. 95, 231105 (2009).

29. Bodrov, S. et al. Terahertz induced optical birefringence in polar and nonpolar liquids. J. Chem. Phys. 147, 084507 (2017).

30. Allodi, M. A., Finneran, I. A. \& Blake, G. A. Nonlinear terahertz coherent excitation of vibrational modes of liquids. J. Chem. Phys. 143, 234204 (2015).

31. Kampfrath, T., Wolf, M. \& Sajadi, M. Anharmonic coupling between intermolecular motions of water revealed by terahertz Kerr effect. Preprint at https://arxiv.org/abs/1707.07622 (2017).

32. Zalden, P. et al. Molecular polarizability anisotropy of liquid water revealed by terahertz-induced transient orientation. Nat. Commun. 9, 2142 (2018).

33. Novelli, F. et al. Strong anisotropy in liquid water upon librational excitation using terahertz laser fields. Preprint at https://arxiv.org/abs/1809.04261 (2018).

34. Zasetsky, A. Y. Dielectric relaxation in liquid water: two fractions or two dynamics? Phys. Rev. Lett. 107, 117601 (2011).

35. Hale, G. M. \& Querry, M. R. Optical constants of water in the $200-n m$ to $200-\mu m$ wavelength region. Appl. Opt. 12, 555-563 (1973).

36. Afsar, M. N. \& Hasted, J. B. Measurements of the optical constants of liquid $\mathrm{H}_{2} \mathrm{O}$ and $\mathrm{D}_{2} \mathrm{O}$ between 6 and $450 \mathrm{~cm}^{-1}$. J. Opt. Soc. Am. 67, 902-904 (1977).

37. Maroulis, $\mathrm{G}$. Hyperpolarizability of $\mathrm{H}_{2} \mathrm{O}$ revisited: accurate estimate of the basis set limit and the size of electron correlation effects. Chem. Phys. Lett. 289, 403-411 (1998).

38. Kalmykov, Y. P. Matrix method calculation of the Kerr effect transient and ac stationary responses of arbitrary shaped macromolecules. J. Chem. Phys. 131, 074107 (2009).

39. Coffey, W. T. \& Kalmykov, Y. P. The Langevin Equation. 3rd edn. (World Scientific Publishing Company, Singapore City, 2012).

40. Boyd, R. W. Nonlinear Optics. 2nd edn (Elsevier, Amsterdam, 2003).

41. Demtröder, W. Laser Spectroscopy: Vol. 2 Experimental Techniques. (Springer, Berlin, Heidelberg, 2008).

42. Levenson, M. Introduction to Nonlinear Laser Spectroscopy. (Elsevier, Amsterdam, 2012).

43. Sekino, H. \& Bartlett, R. J. Molecular hyperpolarizabilities. J. Chem. Phys. 98, 3022-3037 (1993).

44. Palese, S. et al. Femtosecond optical Kerr effect studies of water. J. Phys. Chem. 98, 6308-6316 (1994).

45. Shalaby, M., Vicario, C. \& Hauri, C. P. Extreme nonlinear terahertz electro-optics in diamond for ultrafast pulse switching. APL Photon. 2, 036106 (2017).

46. Sajadi, M., Wolf, M. \& Kampfrath, T. Terahertz-field-induced optical birefringence in common window and substrate materials. Opt. Express 23, 28985-28992 (2015).

47. Ahmed, S., Savolainen, J. \& Hamm, P. The effect of the Gouy phase in opticalpump-THz-probe spectroscopy. Opt. Express 22, 4256-4266 (2014).

48. Amo, Y. \& Tominaga, Y. Low-frequency Raman study of ethanol-water mixture. Chem. Phys. Lett. 320, 703-706 (2000). 
49. Fecko, C. J., Eaves, J. D. \& Tokmakoff, A. Isotropic and anisotropic Raman scattering from molecular liquids measured by spatially masked optical Kerr effect spectroscopy. J. Chem. Phys. 117, 1139-1154 (2002).

50. Jin, Q. et al. Observation of broadband terahertz wave generation from liquid water. Appl. Phys. Lett. 111, 071103 (2017).
51. Gallot, G. \& Grischkowsky, D. Electro-optic detection of terahertz radiation. J. Opt. Soc. Am. B 16, 1204-1212 (1999).

52. Leitenstorfer, A. et al. Detectors and sources for ultrabroadband electrooptic sampling: experiment and theory. Appl. Phys. Lett. 74, 1516-1518 (1999). 